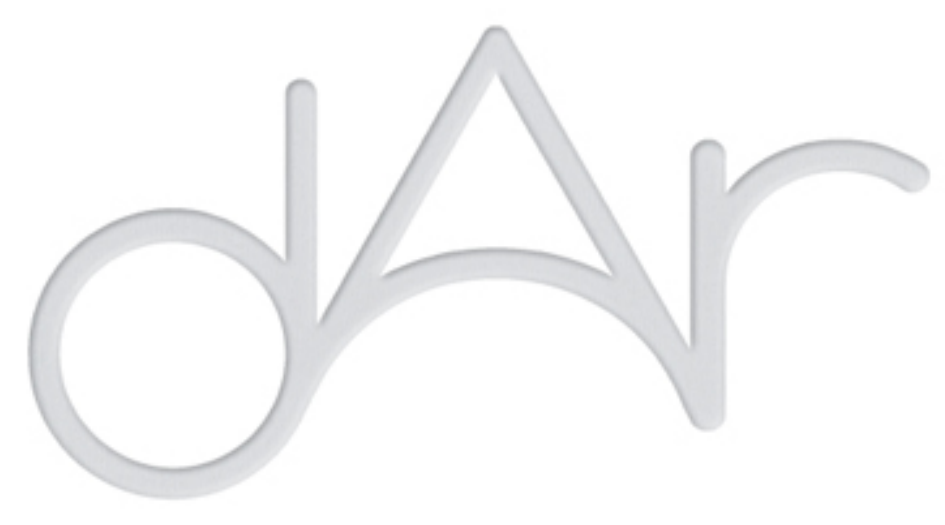

\title{
La valorisation touristique du patrimoine: quelles contributions au développement durable de l'espace fragile des oasis?: le cas des Kasbahs de Skoura Ahl El Oust
}

Autor(es): $\quad$ Oudada, Mohamed; Amara, Lhoussaine

Publicado por: Imprensa da Universidade de Coimbra

URL

persistente: URI:http://hdl.handle.net/10316.2/44436

DOI: DOI:https://doi.org/10.14195/2182-844X_5_15

Accessed : $\quad$ 26-Apr-2023 13:00:36

A navegação consulta e descarregamento dos títulos inseridos nas Bibliotecas Digitais UC Digitalis, UC Pombalina e UC Impactum, pressupõem a aceitação plena e sem reservas dos Termos e Condições de Uso destas Bibliotecas Digitais, disponíveis em https://digitalis.uc.pt/pt-pt/termos.

Conforme exposto nos referidos Termos e Condições de Uso, o descarregamento de títulos de acesso restrito requer uma licença válida de autorização devendo o utilizador aceder ao(s) documento(s) a partir de um endereço de IP da instituição detentora da supramencionada licença.

Ao utilizador é apenas permitido o descarregamento para uso pessoal, pelo que o emprego do(s) título(s) descarregado(s) para outro fim, designadamente comercial, carece de autorização do respetivo autor ou editor da obra.

Na medida em que todas as obras da UC Digitalis se encontram protegidas pelo Código do Direito de Autor e Direitos Conexos e demais legislação aplicável, toda a cópia, parcial ou total, deste documento, nos casos em que é legalmente admitida, deverá conter ou fazer-se acompanhar por este aviso.

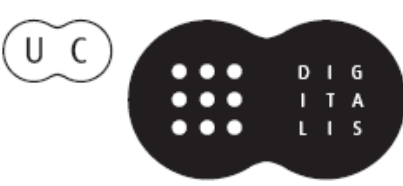



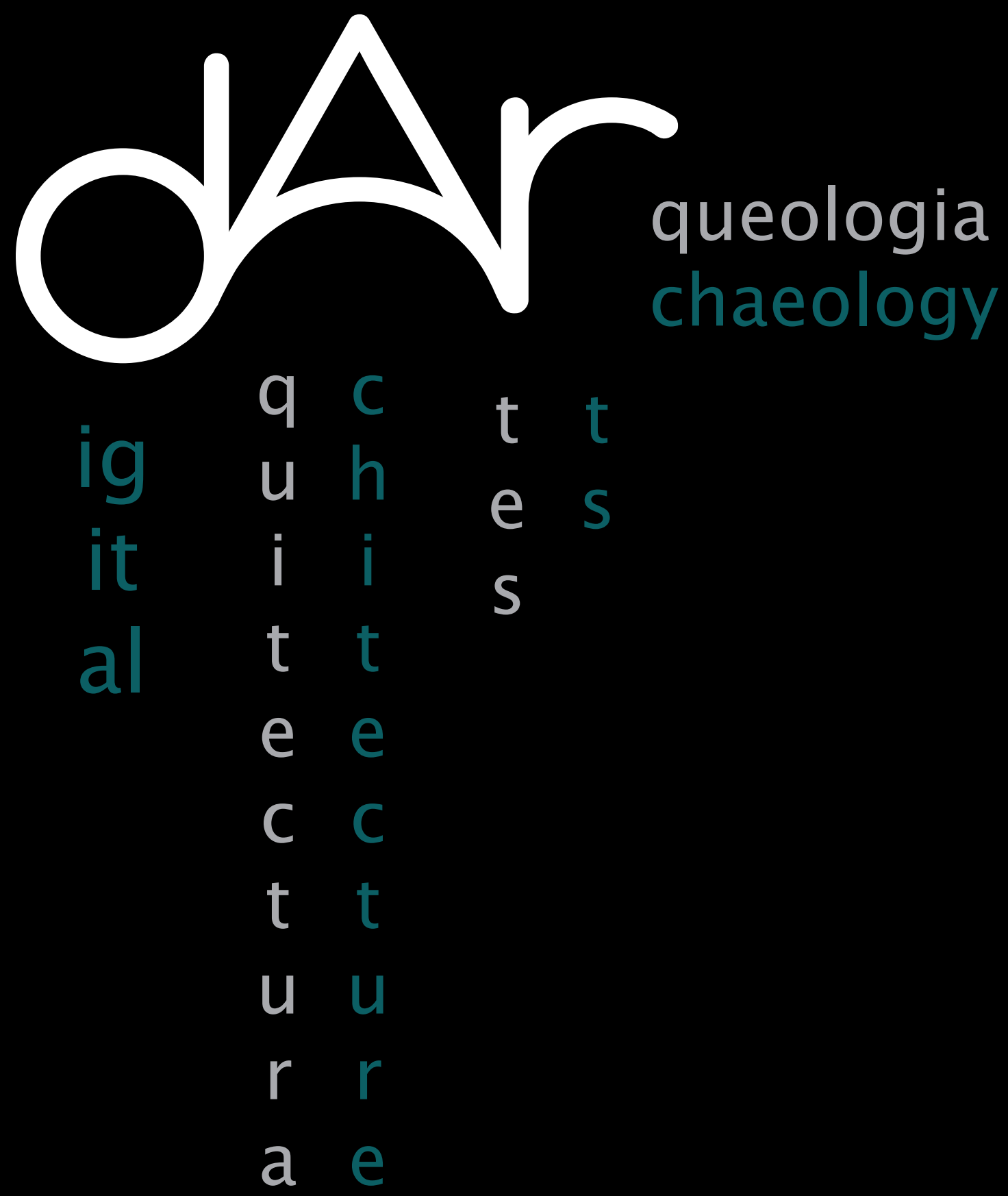

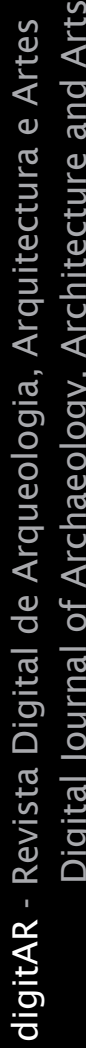




\title{
LA VALORISATION TOURISTIQUE DU PATRIMOINE : QUELLES CONTRIBUTIONS AU DÉVELOPPEMENT DURABLE DE L'ESPACE FRAGILE DES OASIS ? LE CAS DES Kasbahs de Skoura Ahl El Oust
}

\author{
Dr. Mohamed Oudada, Lhoussaine Amara \\ FLSH d'Agadir
}

\section{Résumé}

L'oasis fragile de Skoura dans la Province d'Ouarzazate connaît ces dernières années une dynamique touristique importante.

Cet essor est dû principalement à ses Kasbahs, édifices splendides à base de la terre et hautement riches en valeur culturelle et architecturale.

Ce patrimoine - exposant un état alarmant à tous les égards, perdant son rayonnement d'antan et ne répondant plus aux besoins modernes de la population - est non seulement négligé mais aussi non valorisé en vue d'un développement local fédérateur.

Face à ce constat, nous souhaitons à travers cette communication répondre à la question suivante: Comment, et par quels acteurs, le potentiel économique de ce patrimoine pourrait-il être capable de s'imposer comme vecteur contribuant au développement durable de l'espace fragile oasien à travers la valorisation touristique innovante de ses Kasbahs?

Mots-Clés: Oasis fragile de Skoura, dynamique touristique, Kasbah, développement local fédérateur, acteurs, potentiel économique, valorisation touristique innovante, développement durable.

\section{Introduction}

La question de la contribution del'économie du patrimoine au développement durable des territoires, en particulier des espaces fragiles oasiens et de montagnes, n'est que rarement abordée dans les débats publics au Maroc. L'introduction du patrimoine comme une ressource économique est y encore à ses débuts. Considéré pendant longtemps comme une charge pour l'Etat, le patrimoine, composante essentielle dans la société et objet non prioritaire pour les pouvoirs publics, mais bénéficiaire collatéral des politiques 
et programmes de développement ${ }^{1}$, est aujourd'hui perçu comme une opportunité à saisir, un créateur de richesses et un moyen pour développer ou de renforcer l'image et l'attractivité du pays et ce dans un contexte de mise en concurrence des territoires.

Ce début de prise de conscience de l'importance du patrimoine ${ }^{2}$ et de la nécessité impérieuse de sa valorisation à des fins productives s'inscrivent dans un cadre global caractérisé par le regain d'intérêt pour l'espace local et le rôle, toutefois non négligeable, attribué au patrimoine dans son rayonnement national et international.

Le présent article a pour ambition d'appréhender comment et par quels acteurs, le potentiel économique résultant de la valorisation touristique innovante des Kasbahs ${ }^{3}$ pourraits'imposer comme vecteur de développement durable de l'espace fragile oasien de Skoura ${ }^{4}$.

Pour ce faire, il nous a paru intéressant de tenter d'apprécier l'impact de cette valorisation touristique du patrimoine sur le processus du développement local.

Le choix de l'oasis de Skoura, objet de l'étude du présent article, est justifié par plusieurs raisons :

- la diffusion importante du tourisme, à l'image de son développement dans le sud du Maroc, à partir des années 1990, par la multiplication du nombre d'hôtels, l'évolution de la capacité d'hébergement et le nombre total des nuitées touristiques (Oudada, 2004);

1 ROYAUME DU MAROC, AGENCE ARCHITECTURE HERITAGE ET DESIGN (AAHD), 2010. Diagnostic de l'économie du patrimoine culturel au Maroc, Synthèse et recommandations, p5.

${ }^{2}$ Le patrimoine bénéficie du maillage institutionnel représenté notamment par l'action dans le tourisme (Visions 2010 et 2020), l'artisanat (Vision 2015), l'INDH et le microcrédit. A ce propos, Ph. Aydalot (1985, p.109) écrivait : «c'est dans le cadre local, par la mise en valeur des ressources locales et avec la participation de la population que le développement pourra réellement répondre aux besoins de la population ».Ainsi, « le patrimoine peut devenir une ressource capable de générer des activités. Ces dernières, multiples et variées, mettent en jeu un ensemble d'acteurs et peuvent être un support de réseaux territoriaux » (Landel, 2004).

3 L'approche par la valorisation touristique innovante suscite beaucoup de problématiques qui se rapportent aux processus de valorisation et de développement local fédéré : ressources patrimoniales mobilisées, acteurs impliqués, outils et méthodes utilisés, conditions de mise en place du processus de valorisation et sa durabilité, gestion des incidences occasionnées par ce processus, etc.

4 Les effets socio-économiques et environnementaux de cette valorisation demeurent pendant longtemps insuffisamment évalués voire niés. 
- la présence d'un gisement considérable de kasbahs, représentatives de la richesse culturelle de la zone ${ }^{5}$.

Bien que leur valorisation remette en question la notion de patrimoine, les kasbahs de la région sont transformées en structures d'hébergement de luxe ${ }^{6}$ (Les plus célèbres sont: Amridil, Ait Ben Moro, Ait Abbou, Imadri, Sidi Flah etc);

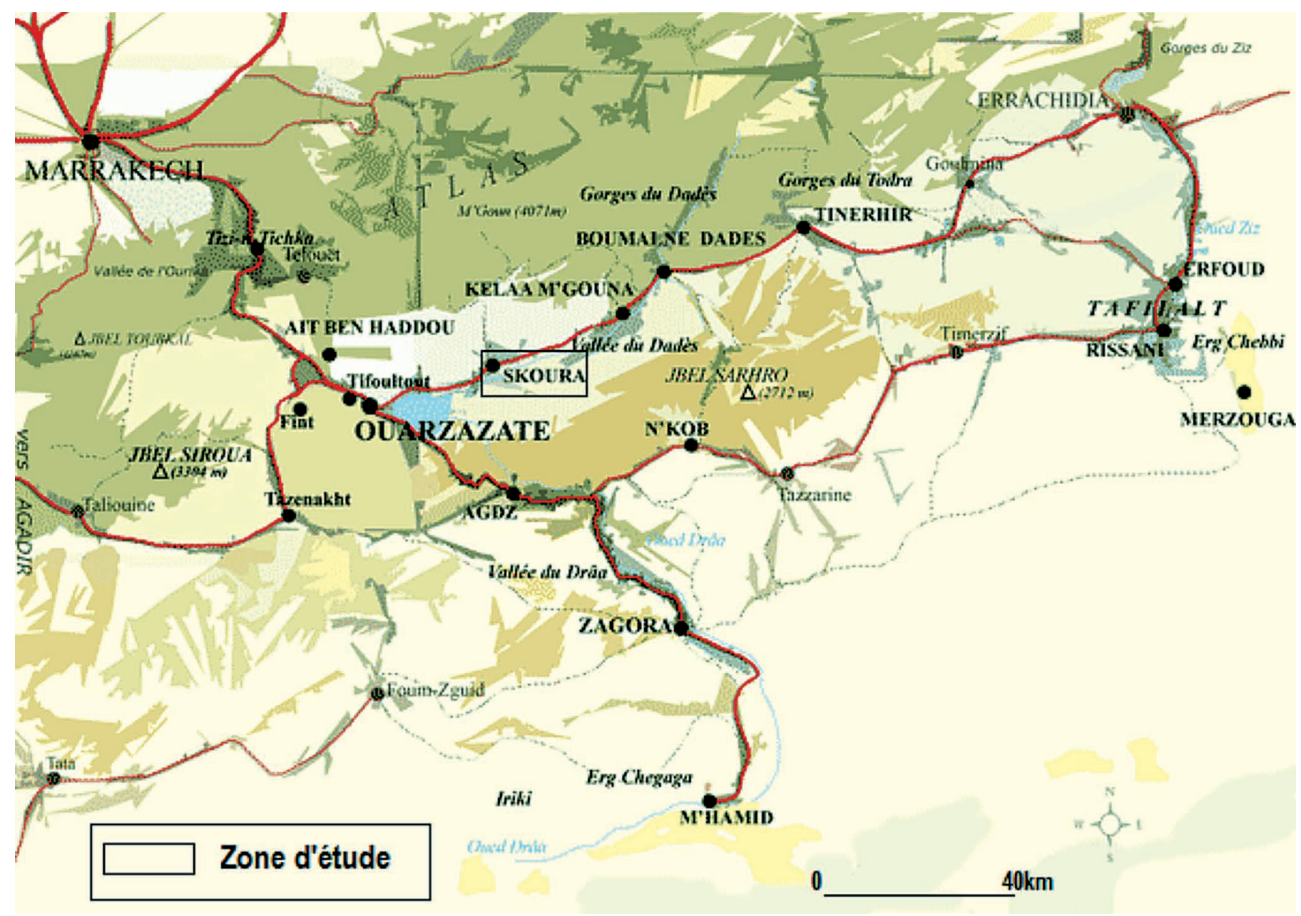

Fig. 1 - Situation de Skoura au Maroc

Source : AMARA, L, 2012, les capacités internes et perspectives de développement d'une commune oasienne, le cas de Skoura Ahl El Oust dans la Province d'Ouarzazate, mémoire du $3^{\text {éme }}$ cycle de l'INAU, p 14.

${ }^{5}$ L'intérêt pour les kasbah et celui accordé à leur valorisation touristique vont de pair. Il s'agit à la fois de créer de nouveaux lieux et produits touristiques afin d'augmenter l'attractivité du territoire et de construire une offre d'activités et par-là créer des richesses et des emplois.

${ }^{6}$ El FASSKAOUI B., 2010. La mise en tourisme du patrimoine architectural dans le Sud-est du Maroc. Quelle authenticité pour quel tourisme ? , dans Jean-Marie Breton, Patrimoine, tourisme, environnement et développement durable, édition Karthala. 
- Le tourisme apparaît comme un espoir de sortie de la crise pour l'oasis dont les activités économiques sont en déclin.

- la population locale est déjà préparée à la mise en tourisme de son territoire. - L'oasis de Skoura, a connu ces dernières années le début d'un développement touristique prometteur.

\section{Présentation de l'oasis de Skoura Ahl El Oust}

Située au Sud-Atlasique du Maroc et faisant partie intégrante du territoire de la région de Draa-Tafilalt, nouvellement créée, et plus précisément de la Province d'Ouarzazate, l'oasis de Skoura relevant de la commune de Skoura Ahl El Oust, fait partie de la région «Atlas et Vallées » selon le découpage régional touristique de la vision 2020. Elle abrite une population de 24055 habitants selon le RGPH de 2014 vivant sur une superficie de $1052 \mathrm{~km}^{2}$.

Elle est située à $40 \mathrm{~km}$ de la capitale provinciale et se compose de douars répartis sur plusieurs fractions dont: Imassine, Ahl El Oust, Amzaourou, Ouled Yaacoub etc.

\begin{tabular}{|l|l|}
\hline Années & $\begin{array}{l}\text { Montant des recettes de la taxe de séjour en } \\
\text { dirhams }\end{array}$ \\
\hline 2013 & 162245 \\
\hline 2014 & 197660 \\
\hline 2015 & 198830 \\
\hline 2016 & 202770 \\
\hline
\end{tabular}

Tab. 1 - Recettes réalisées de la taxe de séjour dans l'oasis de Skoura. Source : D'après les comptes administratifs de la commune de Skoura
La commune oasienne de Skoura Ahl El Oust est administrativement limitée par les communes d'Idelssane et Ghessate à l'Ouest, Toundoute, au Nord, Ait Sedrate Sehl El Gharbia relevant de la province de Tinghir à l'Est et Afla Ndra relevant de la Province de Zagora au Sud.

Cette oasis fragile, est vue comme un espace en difficulté où les contraintes au développement et à l'aménagement sont difficiles: rigueur du climat, rareté de l'eau, etc. Il en résulte donc, un espace qui connaît beaucoup de dysfonctionnements entravant son processus de développement ${ }^{7}$ La

\footnotetext{
7 Sur le terrain un constat fait que cette oasis vit aujourd'hui une situation extrêmement difficile qui s'exprime par les niveaux élevés de pauvreté, de sous emploi, d'exode rural, d'analphabétisme et par de faibles niveaux de scolarisation et d'équipement en infrastructures socio-économiques. A cela s'ajoute l'enclavement et l'éloignement de cet espace des pôles administratifs, universitaires, hospitaliers, commerciaux, et techniques, une seule voie d'accès par le col de Tichka pour un trajet de plus de quatre heures depuis Marrakech sur une route où circulent des voitures, camions, taxis et autres transports touristiques.;
} 
structure économique de la zone se caractérise par sa fragilité : une agriculture vivrière qui souffre énormément de plusieurs problèmes ${ }^{8}$, un artisanat en déclin et un tourisme mal exploité et insuffisamment valorisé ${ }^{9}$ malgré son importance dans l'économie locale en termes de recettes et de nuitées.
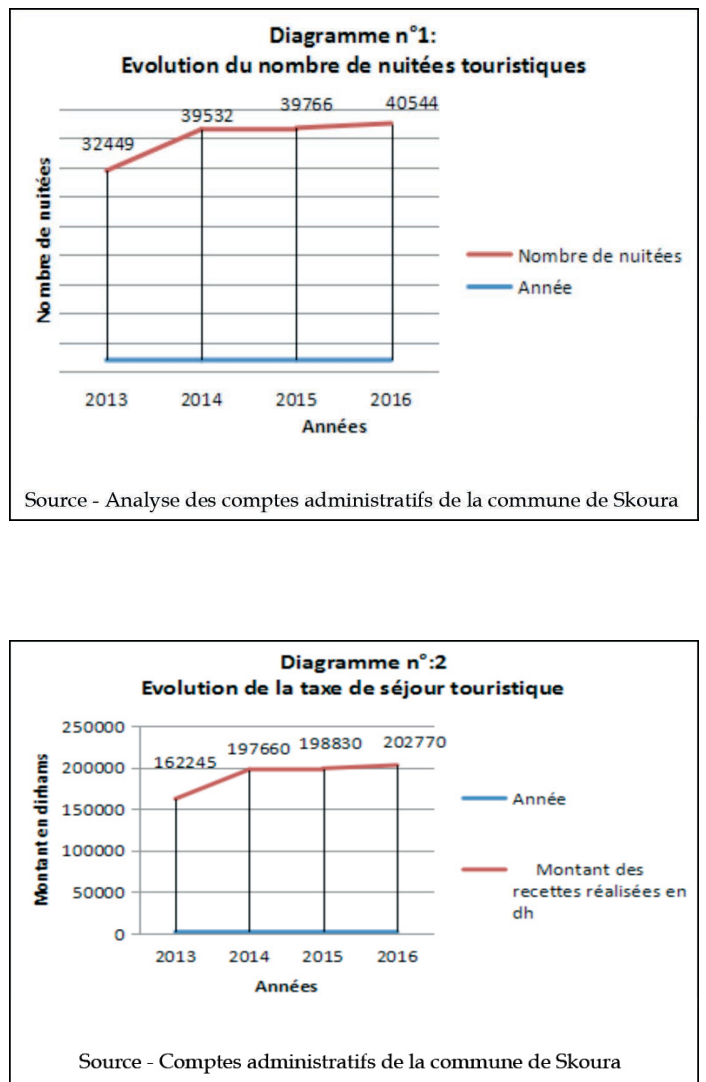

Cette dynamique touristique, l'une des moteurs du développement économique de la région, a une genèse originale appuyée essentiellement sur les kasbahs : patrimoine architectural à base de la terre, hautement riches en valeur culturelle et historique, témoignant d'un art des plus beaux et des mieux adaptés au climat présaharien, conjuguant beauté, confort et originalité .

\section{L'état des kasbahs de Skoura et situation de la population:}

Exposant un état alarmant à tous les égards, perdant leur rayonnement $\mathrm{d}$ 'antan et ne répondant plus aux besoins modernes de la population, les kasbahs, sont non seulement négligées mais aussi non

${ }^{8}$ Celle-ci est confrontée à des multiples problèmes notamment la sécheresse. les conséquences se traduisent concrètement par la faiblesse des rendements, le recul des revenus des agriculteurs, la salinité de l'eau et de sa raréfaction découlant de la gestion irrationnelle du potentiel hydrique, la difficulté de commercialisation des produits due à l'évolution de l'économie de marché, la pression démographique s'exerçant sur l'espace de la palmeraie, de morcellement et de l'abandon des parcelles agricoles, la régression des surfaces agricoles utiles(SAU), le grignotage le mitage des terres agricoles.

9 Il s'agit entre autres d'un tourisme, qui consomme les paysages naturels et culturels, qui profite aux touristes et aux opérateurs touristiques sans pour autant profiter suffisamment à la population locale. 
valorisées en vue d'un développement local fédérateur.

On soulignera également à ce propos que «ce potentiel est doublement pénalisé, d'une part de l'extérieur parce qu'il est fortement méconnu de ceux qui seraient venus justement pour le découvrir et l'apprécier à sa juste valeur ; d'autre part de l'intérieur, parce qu'il n'est pas suffisamment reconnu par les populations et les différents acteurs locaux qui ne font rien, ou peu, pour le valoriser».

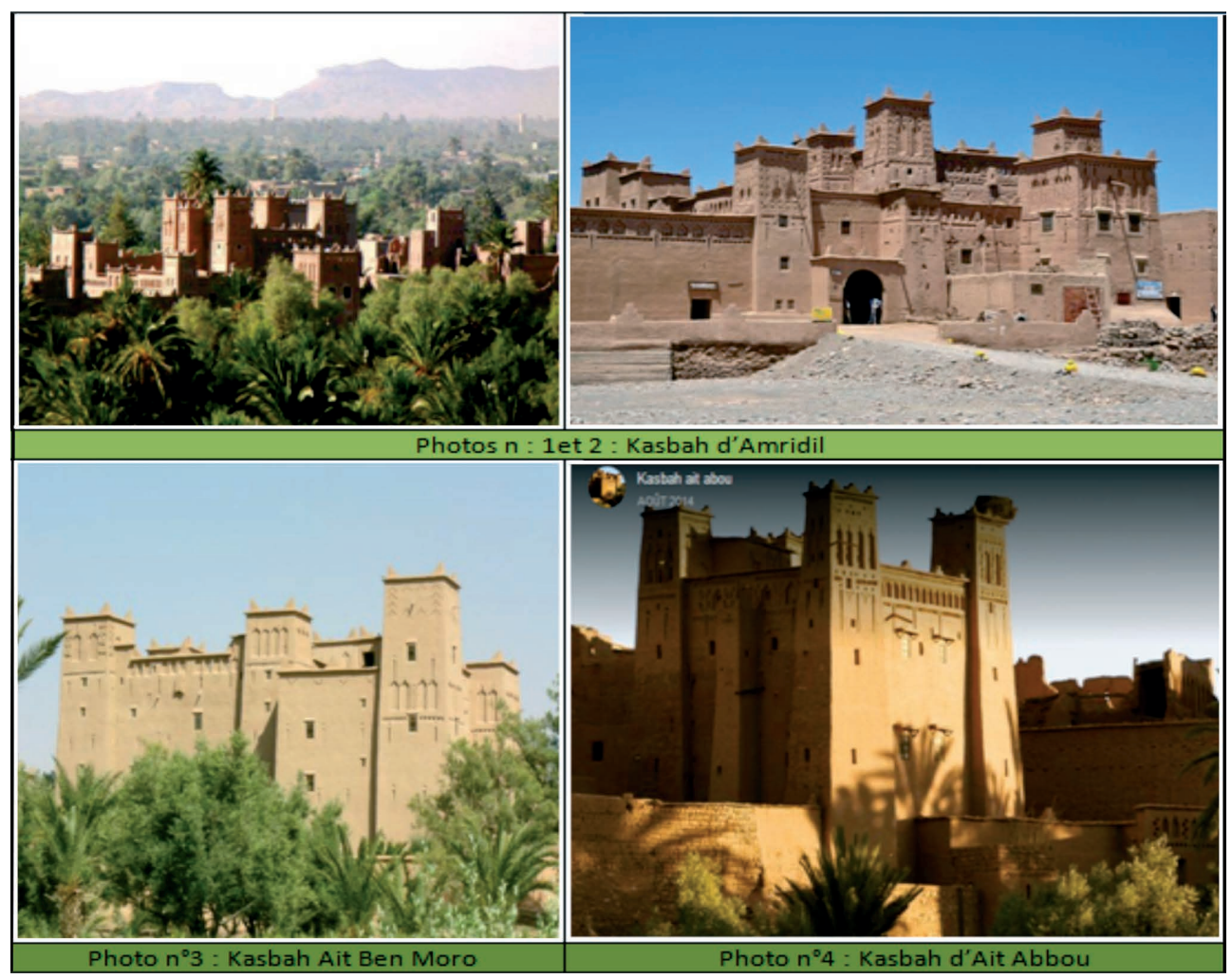

Cette situation de dégradation de ce patrimoine est valable à l'ensemble de la zone présaharienne du pays.

A ce propos, (Popp Herbert, 2008) avance que : " A l'heure actuelle, nous assistons à une situation paradoxale, le produit touristique des Kasbahs est très demandé par les Européens mais il disparait peu à peu. Ainsi, le capital touristique de la région est menacé, car une stratégie publicitaire qui se concentre sur les anciennes kasbahs pourrait échouer si le produit recherché n'existe plus "

Les kasbahs de Skoura sont des demeures individuelles fortifiées appartenant 
généralement à d'anciens notables et qui datent du 17e ou 18e siècle pour les plus anciennes ${ }^{10}$.

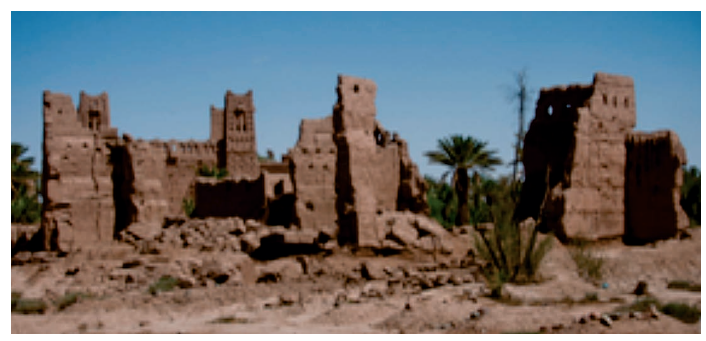

Photo 5 - Exemple de Kasbahs en ruine et abandonnée

Elles constituent un patrimoine tangible (édifices et architecture) et intangibles (pratiques et savoir faire ancestral adapté à cet environnement spécifique qui abrite également des usages économiques).

Elles représentent la mémoire vivante en péril du génie de leurs populations vivant aujourd'hui dans un écosystème de plus en plus fragilisé et menacé d'une disparition définitive, si des dispositions sérieuses ne sont pas engagées en vue de sa protection et de sa valorisation ${ }^{11}$. Les habitants vivent de l'agriculture, de petit élevage et de l'artisanat. Le tourisme et le tournage des films télévisés et cinématographiques constituent deux sources de revenus complémentaires appuyés également par les transferts de l'émigration.

Ces habitants font partie des populations les plus démunies et souffrent: - de la dégradation du bâti : l'état de murailles, des ruelles, des plafonds et des espaces communautaire est fortement dégradé ;

-du manque d'équipements socio-éducatifs, d'accessibilité d'infrastructures notamment en matière d'assainissement liquide, d'accès à l'eau potable et d'infrastructures de base ;

-de chômage et de la faible rémunération du travail artisanal ;

-de la dégradation de l'environnement notamment par la pollution des eaux et de la nappe phréatique due à la faiblesse du système d'assainissement (fosses septiques et puis perdus) et au rejet de certains déchets et eaux usées sans traitement dans les oueds et à ciel ouvert; les effets de la sécheresse et de la désertification etc.

10 La Kasbah d'Amridil date du 17e siècle et a une célébrité manifeste : elle figurait sur les anciens billets de $50 \mathrm{dh}$.

11 Le Maroc et le PNUD ont lancé dernièrement un programme d'accompagnement pour la valorisation durable des ksour et kasbah du Maroc, pour la période 2014-2019. Le plan stratégique (2014-2017) concerne dix sites pilotes, pour un budget total de : 13356020 USD. Aucune des Kasbah de Skoura n'est, à présent, concernée par ce programme. 


\section{1. les causes de la dégradation}

Les causes principales avancées de la dégradation du patrimoine des kasbahs de Skoura se déclinent comme suit :

- Le manque de la conscience et de la sensibilisation de la population à l'impact des facteurs naturels ;

- L'insuffisance des actions de restauration pour des raisons financières ;

- Le manque d'études scientifiques en amont des actions de restauration ;

- Le recul du métier du maalem traditionnel et du savoir-faire,

- Le manque d'un système de communication et de promotion de ce patrimoine,

- L'absence de circuits touristiques appropriés et d'information sur les Kasbahs;

- Le manque de brochures, de guides et de sites Internet inhérents à ce patrimoine ;

- Le manque d'activités génératrices de revenus ;

- La difficulté d'allier le moderne au traditionnel (non adaptation de kasbahs au confort de la vie moderne) ;

- Le mouvement général de migration progressive vers les maisons en dur (dévalorisation des kasbahs par de fortes connotations négatives qui renvoient à la pauvreté) ;

- La disparition des structures sociales communautaires qui permettraient d'assurer l'entretien (absence de l'entraide et l'évolution de la famille patriarcale vers la famille nucléaire) ;

- Absence d'infrastructures de base et enclavement ;

- La faible mobilisation de la commune et les départements ministériels concernés autour de la conservation des kasbahs etc.

\section{2. Les conséquences de la dégradation}

L'ensemble des causes précitées aurait, s'il n'est pas surmonté par la mise en place par les acteurs concernés d'une stratégie claire et volontariste, aura les conséquences suivantes:

-la contribution au déclin du tourisme : le tourisme et les perspectives de son développement sont étroitement conditionnés essentiellement par l'attrait qu'exercent les kasbahs sur les touristes.

La dégradation de cet héritage est de nature à compromettre les chances 
de développement de l'activité touristique ${ }^{12}$.

-l'insécurité par rapport aux constructions menaçant ruine : en effet, la dégradation avancée de ce patrimoine physique met constamment en danger la vie de la population et des visiteurs (éventuels effondrements) ;

-la perte de la mémoire culturelle suite à la disparition de pans entiers du patrimoine (kasbahs ruinées) ce qui constitue la perte et la mort d'une partie essentielle de l'oasis et donc de l'identité culturelle de sa population.

\section{Les kasbahs de Skoura, une valorisation touristique innovante pour un développement durable de l'oasis}

Selon la Stratégie de développement du tourisme rural au Maroc : "L'oasis de Skoura abrite plus d'une centaine de Kasbahs, dont une bonne partie se trouve dans un état de dégradation avancé. (.....) Cependant les touristes sont de plus en plus attirés par le milieu oasien et par l'atmosphère qui se dégage de ces bâtisses en terre. Ces dernières, sont dans un premier temps visitées, pour ensuite servir de structures d'hébergement. 7 unités sont déjà transformées en maisons d'hôtes, gîtes d'étape et hôtel. Plus de 50 sont visitées régulièrement même si elles tombent en ruines, et 4 sont visitées toutes étant habitées. On peut prévoir que dans les années à venir plusieurs d'entre elles seront transformées en hôtels, gîtes, salons de thé, etc. (...). Ces kasbahs, menacées par l'usure du temps seront sauvées si elles font l'objet d'une valorisation par le biais du tourisme. Le séjour touristique sur place qui relaie le passage rapide, est amené à générer des revenus supplémentaires dont ce milieu oasien a grandement besoin (.....)».

\section{1. La valorisation touristique innovante du patrimoine des kasbahs, vecteur de développement durable de l'espace oasien de Skoura? Comment? Par quels acteurs?}

Pour pouvoir répondre à ces interrogations, il nous semble important d'examiner les effets d'entraînement directs et indirects de la mise en

12 La spécificité du cadre esthétique de l'oasis de Skoura est également fortement menacée par les constructions en dur, dans la proximité des kasbah et qui sont souvent bâties sans respect du cadre architectural de la région. Cette situation nuit à moyen et long terme à l'attrait touristique de l'oasis et pourrait engendrer une stagnation ou une baisse d'intérêt des opérateurs et des touristes, et un manque à gagner pour la population de l'oasis en termes de revenus générés par le tourisme. 
tourisme des kasbahs sur l'ensemble du potentiel économique de l'oasis et d'identifier par voie de conséquence les acteurs à l'origine de cette valorisation.

Dans ce sens, A. Bentaleb, avance que : «(...), les effets du tourisme ne sont pas quantifiables et apparaissent contradictoires, de sorte qu'un bilan global est difficile à établir et à apprécier».

Pour A. Bouaouinate (2016, p.54), « la quantification des retombées économiques engendrées par le tourisme au niveau des oasis sudatlasiques du Maroc reste difficile à saisir, vu la complexité des services touristiques ; et pour appréhender l'apport économique de cette activité, on peut distinguer entre plusieurs activités, comme l'emploi direct l' hébergement, le transport, et l'estimation des dépenses des touriste aux oasis visitées(...) encore faut-il poser la question de l'impact économique réel du tourisme sur la population locale des oasis ",

De notre part, nous allons traiter de la relation de cette valorisation, à des fins touristiques, avec les différentes activités à savoir: l'agriculture, le petit élevage, l'artisanat, la production des films télévisés et cinématographiques ainsi que ses implications sur l'emploi, sur la dynamique locale et l'environnement.

\section{1. 1. La valorisation touristique des kasbahs et les activités économiques existantes}

\section{1. 1. 1. La valorisation touristique des kasbahs, l'agriculture et le petit élevage}

Il nous appartient de montrer ici que la valorisation des kasbahs induite par les dynamiques touristiques dans l'oasis a provoqué deux types d'effets sur l'agriculture et le petit élevage.

$\mathrm{D}^{\prime}$ une part, un effet positif dans la mesure où le revenu dégagé des dynamiques induites par la valorisation des kasbahs à des fins touristiques a pu être investi, par certains prioritaires et employés de ces kasbahs, dans les domaines agricole et de petit élevage : achat de nouvelles parcelles agricoles, développement du petit élevage à domicile etc. D'autre part, un effet négatif qui se manifeste par un phénomène de concurrence entre le tourisme et l'agriculture.

Pendant la période touristique, les agriculteurs ont souvent du mal à trouver une main d'œuvre libre nécessaire à l'accomplissement des travaux agricoles. 


\section{1. 1. 2. La valorisation touristique des kasbahs et l'artisanat}

L'activité artisanale, qui se situe au second rang après l'agriculture et le petit élevage, concerne une portion mineure la population de l'oasis.

Avec les travaux de valorisation touristique de certaines kasbahs et le démarrage du tourisme dans la région, cette activité devrait normalement connaitre le même essor qu'a connu le tourisme surtout avec la réalisation de pistes carrossables et la disponibilité des moyens de transport. Or, en réalité, le fonctionnement de cette activité n'a pas pu être en mesure de répondre aux ambitions des artisans de l'oasis.

Cette situation peut s'expliquer par le recours des touristes à la satisfaction de leurs besoins en articles artisanaux dans les villes d'Ouarzazate et de Kelaat Mgouna, voire dans les villes de Marrakech et d'Agadir.

Dans ces villes, l'offre de ces articles est abondante et moins chère.

Il semble également que les acteurs de tourisme notamment les agents de voyages, les guides et les accompagnateurs entrent en connivence avec les «bazaristes » de ces villes pour que la demande soit tournée en leur faveur au détriment des artisans de l'oasis.

A cela, on peut ajouter l'absence d'une organisation de cette activité au niveau local qui pourrait renforcer leur pouvoir et défendre leurs intérêts.

\section{1. 1. 3. La valorisation touristique des kasbahs et la production des films télévisés et cinématographiques}

Le tournage des films télévisés ou cinématographiques dans l'oasis bien que d'une cadence rare génère plusieurs emplois indirects, mais ses effets sur les kasbahs, sont plutôt négatifs du fait de son utilisation abusive, par plusieurs intervenants qui exploitent le flou réglementaire qui entoure l'exercice de cette activité.

Dans le même ordre d'idées, «Les activités liées au tourisme et au tournage de films génèrent certes des revenus supplémentaires mais bouscule les hiérarchies sociales établies et ne profitent pas équitablement à tous les habitants $»^{13}$.

13 ROYAUME DU MAROC (MUAT), 2016 : Plan d'aménagement et de sauvegarde du ksar Ait Ben Haddou, note de synthèse, p4. 


\section{1. 2. La valorisation touristique des kasbahs et la dynamique locale}

Le centre de la commune oasienne de Skoura a connu, avec la diffusion du tourisme induit par la mise en valeur des kasbahs à travers leur réhabilitation et leur reconversion en espaces touristiques d'une grande valeur esthétique et architecturale, un essor économique et une extension urbaine relativement importants.

En effet, les activités qui existaient en nombre limité avant la valorisation touristique des kasbahs, voient leur nombre aujourd'hui augmenter sans cesse.

Nous pouvons citer à titre d'exemple, le nombre de bouchers, de cafés et de commerces des produits alimentaires.

D'autres activités nouvelles ont été créées en rapport avec cette valorisation telles que la procuration des prioritaires des kasbahs valorisées et des accompagnateurs, de véhicules utilisés pour le transport des touristes et d'autres gens de la région ont acheté des fourgonnettes pour le transport de marchandises ${ }^{14}$.

\section{1. 3. La valorisation touristique des kasbahs et l'emploi}

Certes, le déclenchement des travaux de chantiers liés à la valorisation des kasbahs, à travers leur transformation en hôtels, maison d'hôte, musée et centre Équestre, a crée des emplois directs et immédiats pour la population de l'oasis. Il faut noter que ces travaux ont eu des effets positifs sur l'emploi notamment des jeunes. Il n'en demeure pas moins que leurs retombées soient limitées dans le temps.

Les emplois crées sont traditionnellement issus de l'activité d'accueil, d'hébergement, de la restauration, du commerce de souvenirs, des visites etc.

\section{1. 4. La valorisation touristique des kasbahs et l'environnement}

Outre les effets évoqués plus haut, cette mise en valeur touristique des kasbahs se distingue par son impact sur le cadre de vie, élément important

14 - Ces indices témoignent de la dynamique économique qu'a connue le centre de la commune limité auparavant aux activités liées au souk hebdomadaire. 
du développement humain.

Cette qualité environnementale des kasbahs peut s'identifier à travers l'importance de leur composante naturelle et leur qualité esthétique ${ }^{15}$. L'impact environnemental de cette valorisation ne diffère guère, pour l'essentiel, de celui rencontré dans d'autre milieux ruraux ou du littoral.

La fréquentation touristique est à l'origine de prélèvements nombreux et source de déséquilibres de l'écosystème oasien fragile.

En effet, la fréquentation répétée de l'oasis par les touristes non avertis est source d'érosion et d'atteinte aux parcelles cultivées. Un autre problème qui mérite d'être soulevé est celui des déchets solides et d'assainissement liquide, sources imminentes de la pollution de suite à l'absence d'un centre d'enfouissement et de valorisation des déchets et d'une station d'épuration pour le traitement des eaux usées liées à l'activité touristique. A noter, que le traitement des eaux usées au centre de la commune, quant à lui, se fait par l'utilisation des puits perdus, sinon par rejet à ciel ouvert. Si nous prenons en considération la concentration des flux touristiques, le volume des eaux usées augmente pendant la période de la haute fréquentation touristique. L'effet immédiat de cette augmentation sur la nappe phréatique et les cours d'eau se manifeste par leur contamination.

\section{1. 5. La valorisation touristique des kasbahs, quels acteurs à mobiliser?}

Généralement, nous pouvons confirmer que les kasbahs de la zone, n'ont jamais fait objet d'aucune opération de valorisation de la part des acteurs institutionnels ${ }^{16}$.

15 Les kasbahs se caractérisent par leur harmonisation parfaite avec leur environnement naturel et présentent d'importants avantages en matière d'efficacité énergétique et d'adaptation climatique (fraicheur lors de la saison chaude et protection contre le froid en hivers)

16 A noter à ce titre qu'un appel à manifestation d'intérêt, s'inscrivant dans la Vision 2020 et son programme «patrimoine et héritage», a été lancé par la société de mise en valeur des Kasbah (SMVK) et a permis la sélection, parmi d'autres, de la Kasbah Ait Abbou à Skoura. Une convention de sa valorisation touristique a été signée en 2013. Son exécution tarde à voir le jour. Les contraintes rencontrées sont : difficultés liées notamment à la situation juridique marquée par la multiplicité des héritiers et des ayants droit, l'absence d'interlocuteur ayant la qualité de représenter les propriétaires en plus de la situation foncière non assainie de la kasbah.

la SMVK a pour objectif de créer une chaîne nationale d'hôtels aménagés dans des édifices faisant partie du patrimoine architectural et historique du Maroc et rénovés avec des matériaux traditionnels, avec en prime une mise en réseau des différents hôtels de la chaîne dans des itinéraires de découverte explorant les particularités de chaque région en termes de gastronomie, de culture et d'artisanat. 
Les opérations de mise en valeur touristique de ce patrimoine étaient toutefois l'apanage des seules initiatives, étrangères ou autochtones. Plusieurs kasbahs, délaissées, ont été rachetées ou louées et par la suite, restaurées par des promoteurs, en particulier, étrangers, en statut personnel ou en société.

\begin{tabular}{|c|c|}
\hline $\begin{array}{c}\text { Catégorie des unités } \\
\text { d'hébergement }\end{array}$ & Nombre \\
\hline Maison d'hôte & 11 \\
\hline Ferme d'hôte & 03 \\
\hline Gîte d'étape & 03 \\
\hline Auberge & 5 \\
\hline Centre Equestre & 1 \\
\hline Musée & 1 \\
\hline Total & 24 \\
\hline
\end{tabular}

Tab 2 - Kasbahs reconverties en unités touristiques. Source: Bureau des autorisations commerciales de la commune de Skoura

Nombreuses, sont les kasbahs rénovées et reconverties en maisons d'hôte, en musée et en centre Equestre.

En effet, des efforts doivent être consentis pour la sauvegarde des kasbahs de la région et leur mise en valeur par le biais de tourisme. Une plus forte synergie des interventions des principaux acteurs institutionnels (Ministère du tourisme, Ministère de la culture à travers le (CERKAS) ${ }^{17}$, Ministère de l'agriculture à travers l'agence nationale de développement des zones oasiennes et de l'arganier, le Ministère de l'habitat et de la politique de la ville à travers la société Al Omrane, les départements Ministériels de l'environnement, de l'eau et de l'énergie, le Ministère de l'Intérieur à travers l'initiative nationale pour le développement humain (INDH), la collectivité territoriale deSkoura etc.) est de nature à permettre la mobilisation des fonds croisés pour financer les opérations de cette valorisation. La société civile est également un acteur essentiel à mobiliser au profit de la sauvegarde et la mise en valeur des kasbahs, vu son rôle catalyseur pour la mise en place d'un développement local fédérateur. L'implication d'organisations non gouvernementales et d'organismes internationaux à l'instar du PNUD, UNHABITAT et UNESCO est d'une grande importance pour compléter ces efforts ${ }^{18}$.

17 CERKAS, est le centre de conservation et de restauration du patrimoine architectural des zones Atlasiques et subatlasiques. Crée en1989, il a pour objectif, le classement, la protection, la conservation, la restauration et la réhabilitation des ksour et kasbah du sud.

18 UN-HABITAT, fortement impliqué au niveau international dans la conservation des oasis, est déjà intervenu sur le Ksar Ait Ben Haddou et pourra mobiliser son expertise technique notamment en matière de climatologie et de matériaux.

UNESCO, pourra de son côté mobiliser des appuis techniques et appuyer la mobilisation 


\section{Conclusion}

L'oasis de Skoura regorge d'un trésor de kasbahs. Une bonne partie de ce patrimoine architectural a fait l'objet d'opérations de sauvegarde et a été touristiquement valorisée.

A ce titre, L. Amara $(2012, \mathrm{p} 49)$ avance que « ce patrimoine à forte richesse historique attirant les touristes constitue un atout incontournable à même de rehausser la commune parmi les premiers sites touristiques de la région. Mais encore faudrait-il le valoriser davantage et adopter une stratégie innovante apte à promouvoir le secteur de tourisme dans la commune » Ainsi, nonobstant le début de prise de conscience de l'intérêt socioéconomique et environnemental de ces édifices, force est de constater que les opérations de leur valorisation sont encore très limitées au regard de leur potentiel existant.

La sous-exploitation actuelle du potentiel économique en latence de ces kasbahs est de nature à constituer, non seulement, une réserve de croissance pour cette oasis fragile, mais aussi une chance pour améliorer les conditions de vie de sa population.

La mesure de la contribution du patrimoine des kasbahs au développement durable de l'oasis est une mission délicate à mener. Toutefois, cette contribution ne peut aboutir que si nous œuvrons pour la réhabilitation et la restauration de ce patrimoine en vue de sauvegarder la mémoire culturelle de l'oasis.

Néanmoins, ces actions doivent accorder une place centrale au renforcement de la convergence entre les acteurs de développement selon le domaine d'intervention de chacun et au renforcement des capacités de la collectivité territoriale de Skoura afin d'assurer une appropriation de ce patrimoine et mobiliser les ressources humaines et financières suffisantes en matière de préservation ${ }^{19}$ par, entres autres :

- La réalisation d'un inventaire des kasbahs, délaissées et entretenues;

- L'affectation budgétaire spéciale à dédier à la valorisation de ce patrimoine

des fonds complémentaires pour financer les opérations liées à la valorisation touristique des Kasbah.

19 ICHI E., 2007. Les perspectives de développement local d'un modèle des oasis de Tafilalt: le cas de Ghris. Mémoire du 3éme cycle, Institut National d'Aménagement et d'Urbanisme, $331 p$. 
au niveau local et national;

- Assurer et faciliter l'accès des habitants des Kasbahs au crédit bancaire à des conditions avantageuses pour les actions de restauration et de sauvegarde ; - l'adaptation d'un arsenal juridique relatif au patrimoine oasien notamment celui des Kasbahs;

- L'instauration d'un événement médiatique d'une grande envergure pour la collecte des fonds au profit des opérations de restauration et de sauvegarde; - L'encouragement des fonctions d'animation sociale, culturelle et économique en étudiant les opportunités de valorisation (maison d'hôte, restauration touristique, musée, espace artisanal de production et de commercialisation, animation des arts populaires ...);

- La création de circuits touristiques appropriés etc.

\section{Bibliographie}

AMARA, L., 2012. Les capacités internes et perspectives de développement d'une commune oasienne. Le cas de Skoura Ahl El Oust. Mémoire du 3éme cycle, Institut National d'Aménagement et d'Urbanisme, 192p.

AYDALOT Ph., 1984. Economie régionale et urbaine, Economica, Paris

BENTALEB, A., 2013. Impacts du tourisme présaharien sur les ressources patrimoniales dans la vallée de Draa moyen ,Maroc, cas de la palmeraie de M'Hamid, dans DUVAL,M.,PEYRACH GADEAU,V.,OUDADA,M.(dir),Ressources patrimoniales et alternatives touristiques entre oasis et montagne, collection EDYTEM,14,pp.25-34.

BENYOUCEF, B., 2008. Le patrimoine au cœur du tourisme culturel. Colloque International «Tourisme oasien : formes, acteurs et enjeux». Universitée Ibn Zohr, Agadir (Maroc), Faculté Polydisciplinaire d'Ouarzazate, Maroc. <halshs00790476v2>

BOUAOUINATE, A., 2016. La mise en tourisme des espaces oasiens au Maroc, d'un tourisme de masse à un tourisme alternatif, Rapport de synthèse des travaux scientifiques et pédagogiques. Université Hassan II Casablanca ; Faculté des Lettres et des Sciences Humaines Mohammedia, <hal- 01353766>

El FASSKAOUI B., 2010. La mise en tourisme du patrimoine architectural dans le Sud-Est du Maroc. Quelle authenticité pour quel tourisme? , dans Jean-Marie Breton, Patrimoine, tourisme, environnement et développement durable, édition Karthala, ICHI E., 2007. Les perspectives de développement local d'un modèle des oasis de Tafilalt: le cas de Ghris. Mémoire du 3éme cycle, Institut National d'Aménagement et d'Urbanisme, 331p.

LANDEL P.A et PECQUEUR B., 2004. La culture comme ressource territoriale 
spécifique

OUDADA, M., 2004. Désenclavement et développement dans le sud du Maroc : le cas de pays de Bani, thèse de Doctorat de géographie, Université Aix Marseille I, $352 \mathrm{p}$.

POPP, H., 2008. La route des kasbahs, un produit touristique du Maroc présaharien, dans : Minvielle JP, Smida M. et Majdoub W, tourisme saharien et développement durable : enjeux et approche comparative. pp 262-265.

ROYAUME DU MAROC, AGENCE ARCHITECTURE HERITAGE ET DESIGN (AAHD), 2010. Diagnostic de l'économie du patrimoine culturel au Maroc, Synthèse et recommandations, $\mathrm{p} 5$.

ROYAUME DU MAROC, MDF ACHIEVEMENT FUND, 2010. Définition du patrimoine culturel et ses composantes, document consensuel de référence, p5.

ROYAUME DU MAROC, MINISTERE DU TOURISME, 2002. La stratégie de développement du

Tourisme rural au Maroc.

ROYAUME DU MAROC (MUAT), 2016. Plan d'aménagement et de sauvegarde du ksar Ait Ben Haddou, note de synthèse, p4.

ROYAUME DU MAROC (MHPV) et PNUD, 2012. Programme d'accompagnement pour la valorisation durable des ksour et kasbahs du Maroc. 